\title{
Fast timing measurement for the CMS RPC Phase II upgrade
}

\author{
Maxime Gouzevitch* on behalf of the CMS collaboration \\ Univ Lyon, Université Lyon 1, CNRS/IN2P3, IPN-Lyon F-69622, Villeurbanne, France \\ E-mail: mgouzevieipnl.in2p3.fr
}

\begin{abstract}
With the increase of the LHC luminosity foreseen for the High Luminosity phase (HL-LHC) many detectors currently used in the different LHC experiments will be dramatically impacted and some need to be replaced. The new ones should be capable not only to support the high particle rate but also to provide time information to reduce the data ambiguity due to the expected high pileup.

The improved Resistive Plate Chambers using low-resistivity Bakelite are proposed to equip the very forward region of the CMS detector where they can stand rates of few $\mathrm{kHz} / \mathrm{cm}^{2}$. New electronics equipped with excellent timing precision measurement $(<150 \mathrm{ps})$ are being developed to read out the RPC detectors from both side of the strips to allow good spatial resolution along them. The absolute time measurement, determined by RPC signal (around $0.8 \mathrm{~ns}$ ) will also reduce the data ambiguity due to the highly expected pileup at the Level 1 trigger and help to identify Heavy Scalar Charged Particles.

The principle of the measurement, and the implementation of innovative front-end electronic boards (PETIROC front-end ASIC, wave-union TDC and PCB design) will be discussed. First results from test beams at the Gamma Irradiation Facility and the SPS at CERN would also are presented.
\end{abstract}

The 39th International Conference on High Energy Physics (ICHEP2018)

4-11 July, 2018

Seoul, Korea

${ }^{*}$ Speaker. 


\section{Introduction}

In the present CMS detector, the forward region up to pseudorapidity $|\eta|=2.5$ is instrumented by the Cathode Strip Chambers (CSC), while RPC chambers stop at $|\eta|=1.8$. To guarantee a redundancy in this region and improve the muon trigger efficiency during HL-LHC phase, two most external stations of the CMS muon spectrometer would be equipped with improved RPC detectors (iRPC). In this region, the expected rate would rise significantly compared to the actual values, up to $2 \mathrm{kHz} / \mathrm{cm}^{2}$ including a safety factor of 3 [1]. The new stations are required to detect muons with an efficiency above $95 \%$ and to sustain a total integration charge of $1 \mathrm{C} / \mathrm{cm}^{2}$. A good time and space resolution would improve the rejection of background hits and low transverse momentum tracks, and help to resolve ambiguities in the endcap trigger for identification of multiple tracks.

\section{The iRPC project and its characterization}

The classical RPC chambers of CMS are doublet RPC detectors each made of two $2.0 \mathrm{~mm}$ High Pressure Laminate (HPL) electrodes, with a typical bulk resistivity of $1-6 \cdot 10^{10} \Omega \mathrm{cm}$ and separated by a gas gap of the same thickness. The electronics reading those chambers is sensitive to a minimal charge left by a muon of $150 \mathrm{fC}$. They were shown to be operational up to $600 \mathrm{~Hz} / \mathrm{cm}^{2}$, but above their efficiency was declining [2].

Two types of electrodes were proposed to sustain a three times larger rate: aluminum doped glass [3] and modified HPL electrodes. The latter solution was privileged by CMS as a more familiar one with respect to the present RPC system after a first set of tests [1]. A reduction of the electrodes resistivity to $1-3 \cdot 10^{10} \Omega \mathrm{cm}$ and thickness to $1.4 \mathrm{~mm}$ would improve the total evacuation flow of the charge. A thinner gas gap of $1.4 \mathrm{~mm}$ would lessen the produced charge per background hit and limit the stress on the electrodes with effect due to aging.

In consequence, a more sensitive electronics is required to keep the sensitivity high with a reduced pickup charge (see for details Ref. [4]). The signal is readout by a $160 \mathrm{~cm}$ long Printed Circuit Board (PCB) equipped with 48 pickup strips inserted between two HPL gaps. In order to maximize charge induction between the gaps and the strips, we have chosen the minimal thickness of the Flame Resistant 4 (FR4) layers of the PCB compatible with the rigidity and handling capabilities, $300 \mu \mathrm{m}$ on each side of the strips. Two PCBs would equip a full chamber covering an azimuthal region of $20^{\circ}$. The strips of an average pitch of $0.75 \mathrm{~cm}$ are read out from both ends, low radius (LR) and high radius (HR). Both signals are fed into the same Front-End Board (FEB) in such a way that the time difference between them, $\Delta \mathrm{T}=\mathrm{T}_{\mathrm{HR}}-\mathrm{T}_{\mathrm{LR}}$, largely cancels out jitter effects. Subsequently, $\Delta \mathrm{T}$ can be directly related to the along-strip space resolution. The FEB is carrying a fast front-end ASIC, PETIROC2 [5], a 32 channels version developed in SiGe technology. It hosts a preamplifier with a $1 \mathrm{GHz}$ bandwidth and gain of 25 associated with a fast programmable comparator. The comparator output for each PETIROC2 channel is propagated into an Altera cyclone II FPGA hosting the time-to-digital conversion module.

To maximize the signal collected on both ends of the strip, the FEB impedance is precisely matched to the one of the strips. It was observed that this impedance is defined by the distance between the strips and the Faraday cage enclosing the chamber, and is very dependent on the exact chamber design. In the configuration proposed for iRPC it was determined to be $44 \Omega$ [4]. 
Two full size prototypes of the iRPC detector were built in 2017 to test two different ways to connect the PCB to the FEB: using $50 \Omega$ coaxial cables running outside of the Faraday cage (referred to as Coax-scheme) or return strips embedded into the PCB (Return-scheme). It was observed that the Return-scheme prototype is better protected from the grounding and pickup noise than the Coax-scheme one. Both were extensively tested in cosmic rays and then in two sessions of test beams in the Gamma Irradiation Facility (GIF++) [6]. A low intensity muon beam was hitting the chamber together with a uniformly distributed photon background generated by a ${ }^{137} \mathrm{Cs}$ source. During the test beams we had to use a threshold of 81 (108) fC for Return-scheme (Coax-scheme) prototypes. We have verified that amount of electronic noise doesn't exceed $5 \mathrm{~Hz} / \mathrm{cm}^{2}$.
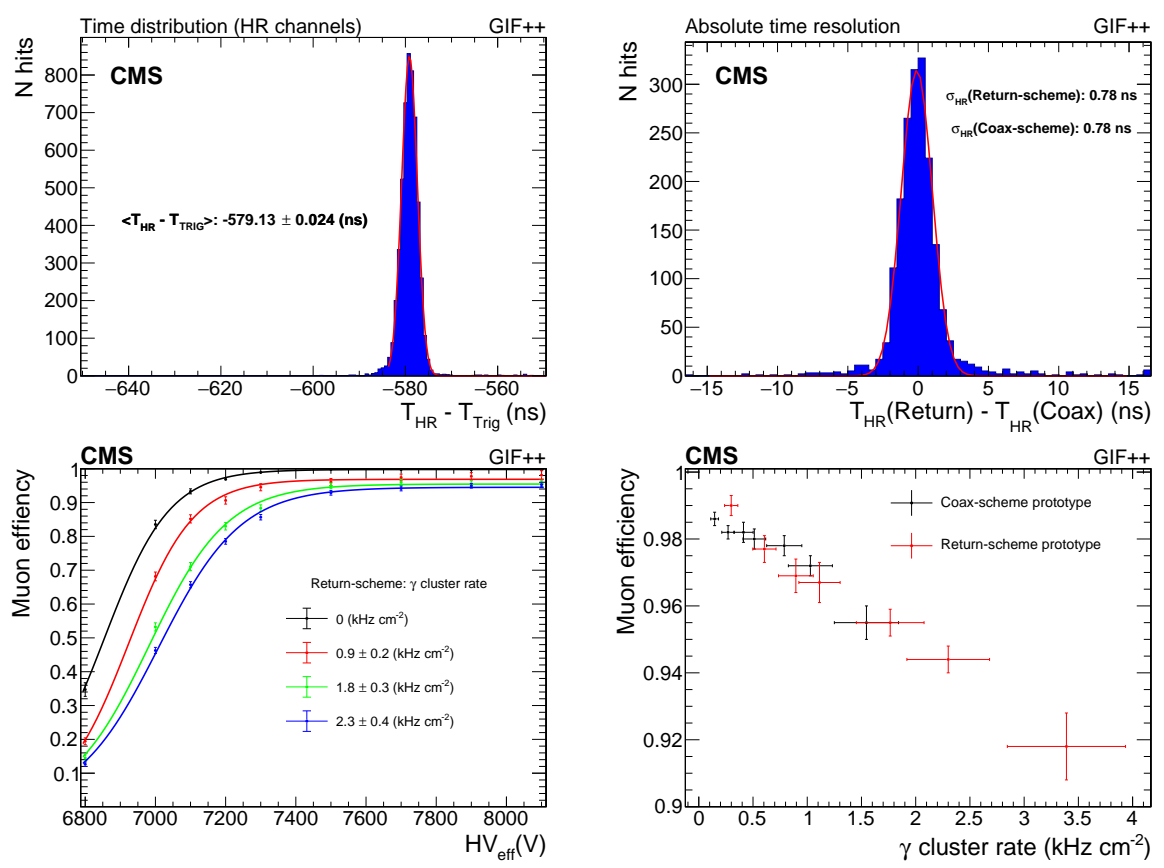

Figure 1: Top.left: time difference between the arrivals of the signal from HR side and from the trigger logic, the red Gaussian fit defines the time jitter. Top.right: difference in time between the HR side of the two prototypes used to measure the intrinsic time jitter of each chamber, $\sigma_{\mathrm{HR}}$. Bottom.left: efficiency curves as function of the effective high voltage for the Return-scheme prototype shown for different background rates. Bottom.right: efficiency at working point for both prototypes at different background rates.

A muon is considered as detected if it was seen by at least one strip from both ends within a time window around the expected arrival of the trigger signal, which is delayed by $600 \mathrm{~ns}$ due to the length of the cables. The size of this time window is defined as $\pm 3 \sigma_{\mathrm{T}}$, with $\sigma_{\mathrm{T}} \approx 2 \mathrm{~ns}$ as shown in Fig. 1.top. This value is defined by a convolution of the trigger jitter, scintillator size and the intrinsic jitter of the chamber. We have measured the latter to be $\sigma_{\mathrm{HR}}=0.8 \mathrm{~ns}$ by comparing the difference of the time of arrival of the signal between the same side of the two chambers. The obtained resolution can be then be divide by $\sqrt{2}$ assuming the chambers electronics to be identical and uncorrelated (see Fig. 1.top). If many strips are localized in space and time, they are gathered together in clusters. The typical cluster size is around $1.2-1.3$ strips, depending on the pitch and background rate, guaranteeing a transverse space resolution much better than $1 \mathrm{~cm}$. 
The presence of muons was also identified by an independent telescope made of 4 scintillators with an effective area well inside the detector acceptance. The efficiency of the chambers was then measured comparing the number of identified muons with those who left a signal in the chamber. Figure 1.bottom shows efficiencies for different rates that are defined as the number of clusters seen by the chamber. We observe that the efficiency of the chamber stays above $95 \%$ up to $2 \mathrm{kHz} / \mathrm{cm}^{2}$ as required by the project specifications. Both prototypes show similar efficiencies. The chamber can be operated at a voltage as low as $7.3 \mathrm{kV}$ for low background and $7.6 \mathrm{kV}$ for maximum rate.

The space resolution was measured with high precision at the H2 line of the SPS complex with two thin scintillators and a special table, produced by DESY, that allows a horizontal and vertical shift of the chambers with a millimetric precision. We observed in Fig. 2 an $\Delta$ T resolution of the order of 130 ns roughly in the center of the chamber that can be converted into the along-strip space resolution $\Delta y \approx 1.3 \mathrm{~cm}$ using the formula $\Delta y=v \cdot \Delta \mathrm{T} / 2$. The speed of the signal propagation $v$ was measured looking at the shift of $\Delta \mathrm{T}$ in ns when the DESY table is moved by $y \mathrm{~cm}$ (see Fig. 2).
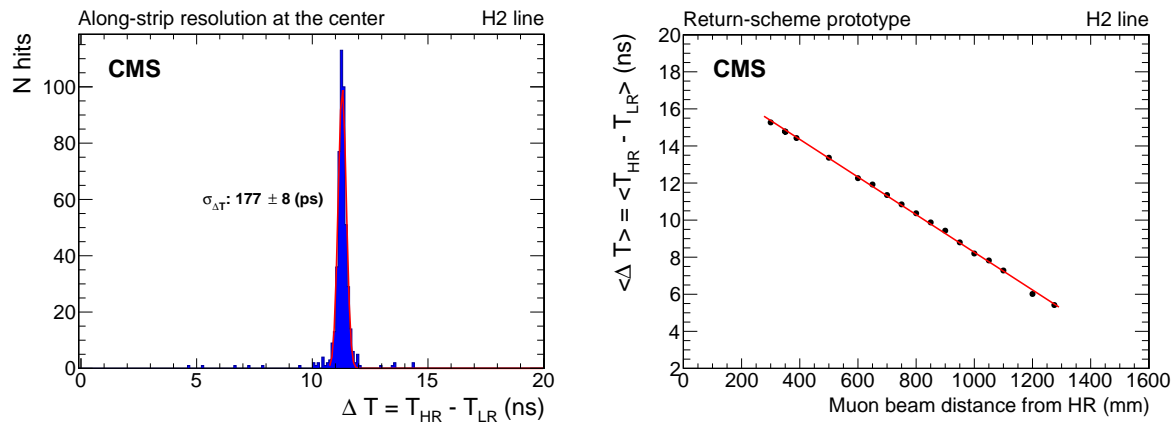

Figure 2: Left: $\Delta \mathrm{T}$ resolution of the Return-scheme prototype at the center of the detector. Right: correlation between the average $\Delta \mathrm{T}$ value and the position of the beam with respect to the HR.

\section{Summary}

The iRPC upgrade project was validated in a test beam and fulfills the requirements of operation in the high background rates expected during HL-LHC phase: efficiency above 95\%, space resolution at $\mathrm{cm}$ level and absolute time resolution better than $1 \mathrm{~ns}$.

\section{References}

[1] CMS Collaboration, The Phase-2 Upgrade of the CMS Muon Detectors, Tech. Rep. CERN-LHCC-2017-012. CMS-TDR-016, CERN, 2017.

[2] CMS Collaboration, The CMS experiment at the CERN LHC, JINST 3 (2008) S08004.

[3] M. Gouzevitch et al.Nucl. Instrum. Meth. A 845 (2017) 226 [1606 . 00993 ].

[4] C. Combaret et al., Fast timing measurement for cms rpc Phase-II upgrade, JINST 13 (2018) C09001.

[5] J. Fleury et al., Petiroc, a new front-end asic for time of flight application, in 2013 IEEE Nuclear Science Symposium and Medical Imaging Conference (2013 NSS/MIC), pp. 1-5, Oct, 2013, DOI.

[6] M. R. Jakel et al., CERN-GIF++, PoS TIPP2014 (2014) 102. 